\title{
The Use of NSVC and Their Effects on SRL, Learning Strategies, and Oral Production
}

\author{
Ayyub Abdurrahman \\ Islamic State University Ar-Raniry, Banda Aceh, Indonesia \\ E-mail address: ayyubar070@gmail.com
}

\begin{abstract}
Keywords: EFL Student, oral skill, active vocabulary, Native Speaker Video Clips with Subtitles (NSVCS), Self Regulated-Learning (SRL).
\end{abstract}

\begin{abstract}
This study investigated the use of Native-Speaker Video Clips (NSVC) to teach oral skills in English. A student with medium achievement in English was selected and given the treatment that comprised 12 weeks of recorded practice interspersed with 6 fortnightly oral presentations to the writer that were also recorded. The practice clips were analysed for the types of learning strategies employed, namely rehearsal, elaboration, and organization, and SRL which included meta-cognition, motivation and perseverance, while the oral production recordings were analysed for fluency, pronunciation, stressing, intonation and understanding. The findings showed that she adjusted the levels of use of almost all of HSRL factors following the feedbacks after each oral presentation except elaboration and organization factors, and those oral production skills were improved gradually. The findings revealed that it was purposeful to remove code-switching tendencies in the student.
\end{abstract}

\section{INTRODUCTION}

Generally, there was a universal failure in the teaching of the oral components of English by non-native instructors whose incompetence caused code-switching to be quite frequent during the teaching process. The language comprised four skills, namely, listening, speaking, writing, and reading, however, the emphases in the classrooms were on grammar, vocabulary and formal sentence structures in writing and reading as found in textbooks. Also, evaluations of mastery and comprehension were also based writing and reading using paper and pencil tests that employed multiple-choice items, cloze tests, sentence completions tasks or dreaded essay questions. There were the occasional oral or listening tests but their contribution to the total achievement score was negligible.

Together with policy makers, non-native instructors had also adopted the view that the best way to teach the complete English was to send students abroad or to immerse them in a community of native speakers such as that found in an English international school. This strategy had been shown to work extremely well but it was not realistic or affordable for many students in developing countries.

\section{LITERATURE REVIEW}

\subsection{An Overview of L1, L2 and L3 Learning}

Another shortcoming in the teaching of English as a second (L2) or foreign language (L3) was the many competing theories and models that attempt to explain Second Language Acquisition (SLA). Also, the teaching of a L2 to adults was very different than that of childern. Though, the SLA theories did not differentiate into theories language learning for kids and theories for adults. One theory suitable for kids was Chomsky's (1965) universal grammar theory where grammar of language defined as a version of the structure capability of the inborn spokesmans' knowledge tacit of their language. It was considered in some criterion; a) The universality, b) Descriptive adequacy, c) Had to be qualified in nature, d) Could be easily learned. In other word, grammar had to be as simple as possible. Universal Grammar also had integrated into numerous language special variants structure that kids ought to master in acquiring their native language. 
Vygotsky however (in Min, 2006) believed that learning of a new language was embedded in social interactional, civilizing, institutional, and chronological context. All the actions in L2 learning showed the social interactional characteristics that embedded in a social interactional context. In his view, social interaction not only considered about a context but also caused L2 development. Though, communal communications did not guide promptly to L2 improvement but by the student's psychological processes. In this process, individuals L2 competence was shaped greatly depended on the way they transmitted of social communcations. Language improvement result shaped from the frequency, quality and level of social interactions itself which included the direct conversational and indirect interaction.

The cognitive perspective extended the SLA theories to adults. Krashen (1978) stated that input was the greatest sole determiner of language aqcuisition and an input could be made meaningful through code-switching strategies, i.e., the use of features of L1 to assist understanding and mastery of L2. Code-switching mechanisms were explained using schema theory which stated that the schema was the data structure for representating the concepts stored in memory for generalizing the concepts underlying object, situations, events, sequence of events, actions and sequences of actions (Rumelhart \& Norman,1983). There were four essential characteristics of schemata, which combined that made the powerful for representing knowledge in memory, such as factors, could join one another, common ideas and the knowledge. Code switching might be acceptable for conceptual understanding but it had been shown to be harmful for oral production. Krashen further stated, outcome had no straight function in language gaining. He also stated that good linguistic capability was probable with no outcome and there was no direct proof that graspable outcome guides to language aqcuisition. However, it was strongly proved that input alone was not sufficient enough to reach high levels in SLA.

Contrary to Krashen's input, Swains (1993) said that output might lead directly to language aqcuisition, which she also specified four ways of output that might play a role in process in L2 learning: a) to develop fluency and automaticity in language function; b) to allow students detect what they understand incompletely or not; c) to provide them chance to test innovative illustration; and d) to create advice which can guide them to adjust their outcome. Consistent with this view was Bandura's (1986) social cognitive theory which included three factors, namely, Environment, Personal and Behaviour that had been argued to shape the learners in their SLA.

Ullman's (2001), planned a technical style with the technical recall style underlying the phsychological glossary while the technical style supported elements of psychological structure. Technical recall was a subordinate recall that pilled up not only thruths and occasions, but also lexical understanding, including the sounds and senses of vocabulary. Mastering innovative vocabulary strongly based on medial sequential lobe constructions. Ultimately, the understanding of vocabulary turned up to be autonomous of the medial sequential lobe and needy on other neocortical areas, principally those in sequential and temporoparietal areas. The sequential lobe might become principally vital for piling up vocabulary senses, while temporoparietal areas might become more significant in pilling up vocabulary sounds. Lexical recall was not informationally summarized, but was easy to get to numerous psychological style.

Compounding competing theoretical positions were the techniques of teaching that were believed to be supported by strong theories or a mixture of theories. Instructors were justified by citing Krashen, Bandura, or Rumelhart \& Norman and their associates, while the use of peer or cooperative support was leaned on Vygotsky. The use of mediated instruction such as ComputerAssisted Langauge Learning (CALL), audio recordings and other similar facilities whether individually or with cooperatively were welcomed as it overcomes the weaknesses of the instructor method especially in the aspects of providing the required exercises, repetitions, feedback, and assessment on behalf of the instructor. The eclectic theoretical positions and technical executions, however, had not improved the efficiency of mastery of English by foreign students.

\subsection{Unobservable Learning Process}

Over the last 50 years, the teaching of English had taken a dramatic cognitive slant where the instructor provided the inputs and tasks to match the presumed cognitive capabilities of the adult 
students. What had been overlooked was that adult students might still have in them the abilities to learn another language using the same mental techniques and strategies they had employed unconsciously when learning their mother tongues. These abilities might have been subsumed or buried deep under their current cognitive and meta-cognitive abilities to the point that when the learning tasks were simple or superficial they were not invoked or reactivated.

Levelt (1998) offered a model of learning based on the L1 processes. According to Levelt, the process of language learning was not simply of mimicking or modelling to reproduce the sounds or phonetic strings. Continuous exposure to a language helped the students to develop their internal speech in that language through a series of mental tasks such as parsing, conceptualizing and formulating together with developing conceptual understanding and meanings for the words and sentences, they also developed internal structures in the form of phonemization, speech motor patterns and articulatory motor systems that allowed for a reconstruction or reproduction from the learned phonological inputs received, whether in the form of words or sentences were not acquired whole. They were deconstructed into forms of protosyllables to be reproduced or differently reconstructed following the L1 protosyllabary and phonemization techniques until lexical concepts, semantic structures, and syntaxes of the target language (L3) were acquired. The processes were graphically presented in Figures 1.

According to Levelt, there were two levels of processing in the learning of a language, namely, the internal or mental level that involved the creation of internal speech, and the physical or oral level that involved the production correct sounds and tip of the tongue fluency. Thus, students might appear to model or rehearse a word or sentence, but in reality they were build internal structures of the new language, namely, speech motor pattern and articulatory motor systems through the process of phonemization. As the goals of teaching an L2 were the acquisition of internal speech and correct oral production in the L3, authentic speech or native-speaker oral presentations were crucial.

\section{PROBLEM STATEMENT}

Watkins and Wilkins (2011) and Potosi et al (2009) explored the effects of using YouTube videos in EFL classroom and outside the classroom and found that they were effective in improving conversation, listening, and pronunciation skills. The use of video clips outside the classroom on a systematic basis required the students to employ various learning strategies specific task of learning a new language and also continuously monitored, modified, and regulated all the necessary actions towards achieving the goal of full mastery of the new language. The importance of Self Regulated Learning (SRL) in language learning and in technology enhanced environment that had been shown by many studies (Huang, 2013; Steiner et al, 2013). Jayakumar (2009) further argued that influential measurement was the recipe component in SRL and drove continue use of the video clips. Nicol \& MacFarlane-Dick (2006) proposed a model that incorporates all the necessary factors for self-learning using video clips (Figure 1). Many studies had investigated the effects of video lessons in language learning, the use of learning strategies, and the importance of SRL but no study had investigated how the goals were set by the instructor in the beginning of a session (native like) and the feedback presented at the end of the Classroom Oral Production (COP) session affect students modifications of their learning strategies and SRL through subsequent sessions. Thus, this study investigated the use of Native-Speaker Video Clips (NSVC) in teaching English oral skills outside the classroom and its effects on SRL and performance.

\section{RESEARCH QUESTIONS/PURPOSE OF THE STUDY}

Live inputs by native speakers of English were difficult to find in poor developing countries such as Indonesia. As an alternative, this study employed recordings of native speakers that were easily available on the web. The recordings should work equally well if they contained all the elements proposed by Levelt and the students engaged through the elements as they interacted with the recordings. The use of video recordings allowed students to work at their own pace and 
employed the set of learning strategies they felt appropriate for the tasks at hand. As students engaged individually in viewing the recordings, they would be driven by their SRL strategies that encompassed meta-cognitive, motivation and perseverance strategies.

Based on the above discussion, the focus of the study was to determine:

- The learning strategies and SRL strategies employed by the students, and

- Whether there were improvements to oral performance in terms of motivational beliefs, student goals, learning strategy, SRL and duration and frequency in activating the students' oral vocabulary.

\section{METHODOLOGY}

\subsection{Participants}

23 EFL students from a two-credit Vocabulary and Pronunciation course of 2013 participated in this study.

\subsection{Procedures}

The students were assigned by instructor as the writer to watch videos and to familiarize themselves with them for about 20 hours over the period of 2 weeks for each topic covering areas they were familiar with, namely Islam, Animals, Humans, Sports, Agriculture and Economics, based on the syllabus given by the English Department of Tarbiyah Faculty of UIN Ar-Raniry. They were allowed to choose and download clips they liked freely whose narrators were restricted to British or American presenters only. They were required to record themselves each time they viewed the video during their Home Self-Regualted Learning (HSRL) practice.

At the end of after two weeks of one HSRL practice for one topic and the beginning point for the next HSRL topic, they had to submit their HSRL practice recordings and present their $\mathrm{COP} /$ monologue to the instructor in the presence of their friends which was also recorded by him, after which they were given frank feedback in a dynamic, personal and supportive way regarding their strenghts and weaknesses observed during their presentation.

Furthermore, the evaluation was done during COP taking place based on story telling and dialogue. He would interject of any common mistakes they made and corrected them with detail corrections, solutions, suggestions and appreciations on the factors they were already good in smilingly, fairly, patiently and softly that they noted, to later on, used to improve their strategies in their coming HSRL practices and provided with assessments of their performance immediately and transparently. Eventhough, they felt stressed in first and second round any way, but not again in the following ones.

This treatment, the formative assessment and SRL strategy, lasted for 16 weeks during in which they were treated fairly and with respect to avoid making them feel threatened, judged, or intimidated, especially in presenting their COP. This approach was successful in making them feel relaxed and conversational. The aim was to enhance their vocabulary acquisition, especially in aspects such as fluency, pronounciation, stressing, intonation and understanding.

The procedure of the study follows Figure 1.

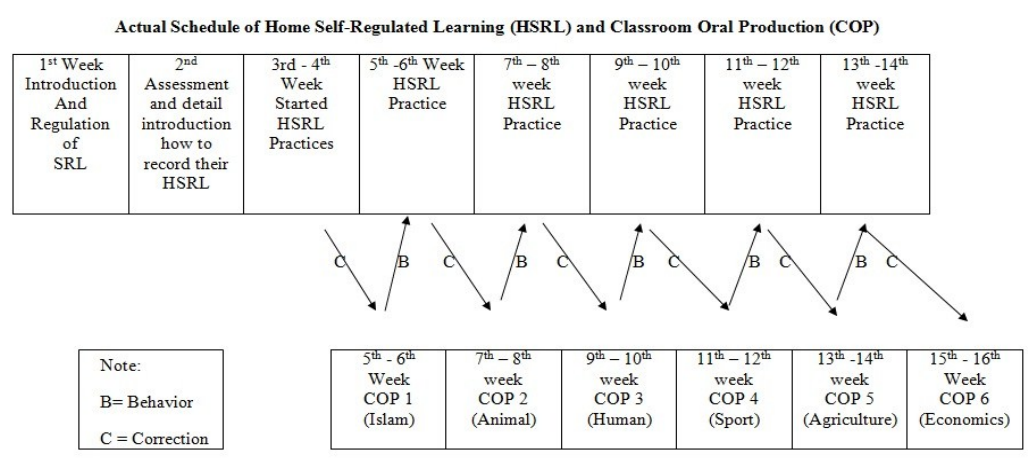

Figure 1: Procedures of the study 


\subsection{Instruments}

This study followed the combination prescriptions adapted from both Levelt (1998) and Nicol \& MacFarlane-Dick (2006) to support and develop the students' self-regulation during their individual engagements with the NSVC and the instructor (Figure 2). However, it reported the findings related to one student only who was at the medium level of achievement as the first pilot project of the writer.

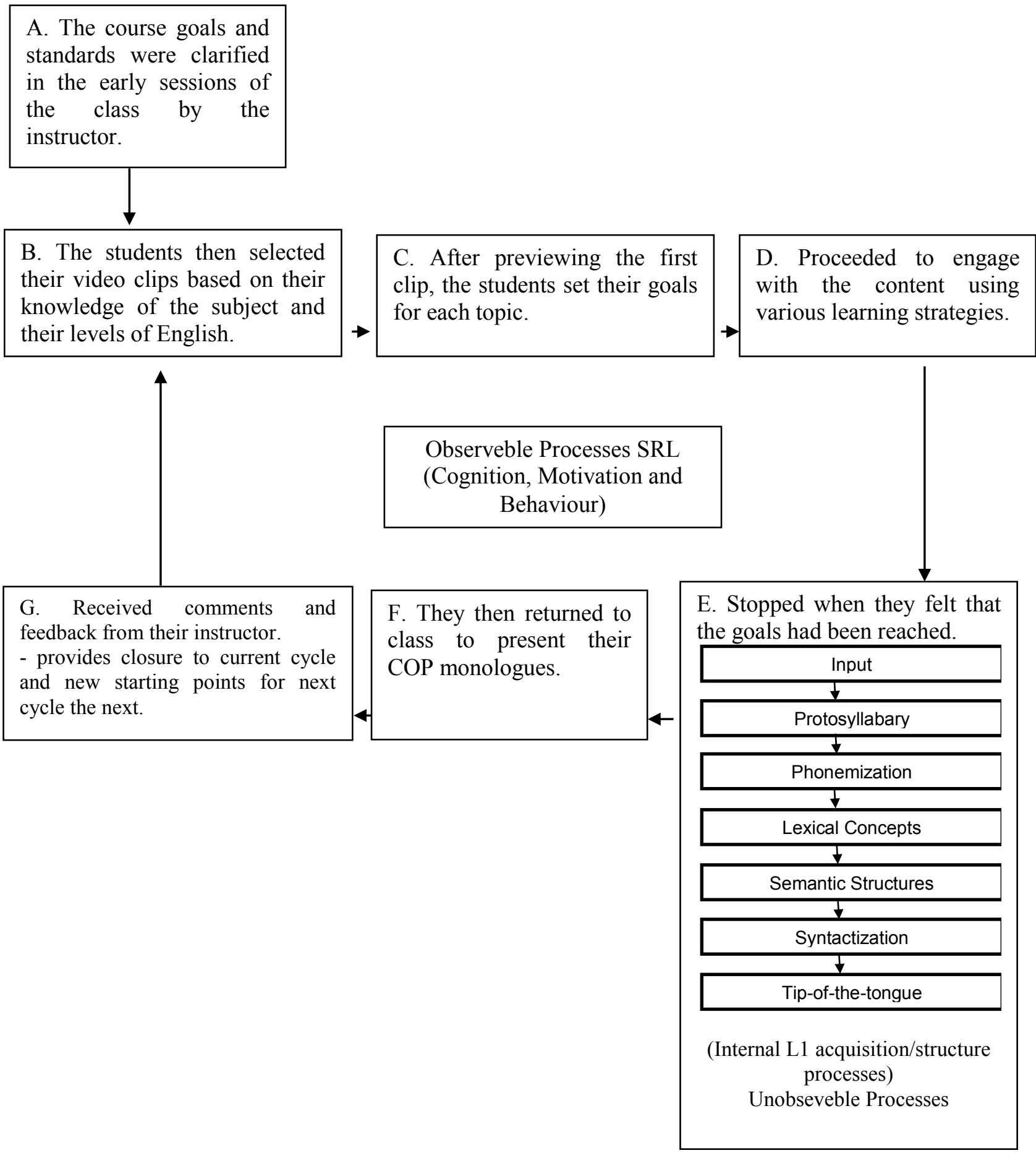

Figure 2: Adapted SRL Nicol and MacFarlane (2006) with Internal L1 acquisition/structure processes Levelt (1998). 


\subsection{Data Analysis Procedure}

The data, the use of NSVC, SRL and learning strategies, which was based on the improvement of oral vocabulary made, in HSRL Practice and COP records analysis was conducted through the following steps: a. classifying data, b. typing the transcipts, c. cross-checking the transcipts, $d$. coding the trancripts, e. analyzing the coding based on the scale of the rubric strategy, f. putting the result into tables and figures, and g. making conclusions by comparing the oral error reduction progress of the student.

It was assumed that it would be contributed by the acquisition of new knowledge and skills and the reduction of errors that would proof the number of code-switching reduction as a proof of success in activating oral vocabulary and it was done by the instructor, 2 best students, 3 best alumnies and 3 senior English speaking instructors who had good mastery and high sensitivity in English pronunciation factors of English Department of UIN Ar Raniry.

Analysis of HSRL. There were three transcripts of HSRL Practice records analyzed. The first one was Islam topic that made and recorded at week 3 and 4, the second one was Sport topic taken from week 9 and 10 and the last one was Economics topic taken from week 13 and 14 and assumed to be able to see how the student controlled herself in her HSRL practice, as follows:

- HSRL factors coding system included three parts and were coded by: 1. Rehearsal: (...), 2. Motivation such as developing pronunciation: $[\ldots]$, developing understanding: $\{\ldots\}$, not clear pronunciation for one word: /.../ and not clear pronunciation for phrase or sentences: //.../ and 3. Perseverance/patience: $<\ldots>$.

- HSRL factors scale analysis was done based on the varied errors made by the students. It was normalized into the modified model rubric suggested by Dannelle D. S., \& Antonia, L. (2005).

Analysis of COP. It was done by analyzing the transcripts of 6 topics COP performance records which was assumed to be contributed by the acquisition of new knowledge and skills and the reduction of errors in order to be able to see the code-switching reduction.

- COP factors coding system included three parts and were coded by: 1. Fluency: <...>, 2. Pronunciation: /.../ and 3. Stressing: '.

- COP factors scale analysis was done based on the varied errors made by the students. It was normalized into the modified model rubric suggested by Dannelle D. S., \& Antonia, L. (2005) to avoid decimal point the maximum scale for each COP factors was marked 20.

\section{FINDINGS}

This study reported the findings from one student that covered 9 of 31 HSRL practice recordings covering 7 hours 26 minutes 19 seconds. Other 6 COPs ones covering 13 minutes 15 seconds were analysed. 
Table 1: HSRL Factors Result Scale

\begin{tabular}{|c|c|c|c|c|c|c|c|c|c|c|c|c|}
\hline \multirow{2}{*}{ No } & \multirow{2}{*}{\multicolumn{3}{|c|}{ HSRL Factors }} & \multicolumn{3}{|c|}{ Islam } & \multicolumn{3}{|c|}{ Sport } & \multicolumn{3}{|c|}{ Economics } \\
\hline & & & & 1 & 2 & 3 & $1^{*}$ & 2 & 3 & 1 & 2* & 3 \\
\hline \multirow[b]{3}{*}{1} & \multirow{3}{*}{$\begin{array}{c}\text { Domain } \\
\text { knowledge/ } \\
\text { strategy } \\
\text { knowledge/ } \\
\text { motivational } \\
\text { beliefs }\end{array}$} & \multicolumn{2}{|c|}{ Variety } & \multicolumn{3}{|c|}{100} & \multicolumn{3}{|c|}{80} & \multicolumn{3}{|c|}{80} \\
\hline & & \multicolumn{2}{|c|}{ Difficulty } & \multicolumn{3}{|c|}{100} & \multicolumn{3}{|c|}{80} & \multicolumn{3}{|c|}{100} \\
\hline & & \multirow{2}{*}{\multicolumn{2}{|c|}{ Passage Length }} & \multirow{2}{*}{\multicolumn{3}{|c|}{100}} & \multirow{2}{*}{\multicolumn{3}{|c|}{80}} & \multirow{2}{*}{\multicolumn{3}{|c|}{80}} \\
\hline 2 & $\begin{array}{c}\text { Student } \\
\text { Goals/Contents }\end{array}$ & & & & & & & & & & & \\
\hline \multirow{3}{*}{3} & \multirow{3}{*}{$\begin{array}{l}\text { Learning } \\
\text { Strategy }\end{array}$} & \multicolumn{2}{|c|}{ Rehearsal } & $<\mathbf{5 0}$ & $<\mathbf{5 0}$ & $<\mathbf{5 0}$ & $<\mathbf{5 0}$ & 100 & $<\mathbf{5 0}$ & 80 & $<50$ & 80 \\
\hline & & \multicolumn{2}{|c|}{ Elaboration } & $\mathbf{0}$ & $<\mathbf{5 0}$ & $<50$ & $\mathbf{0}$ & $\mathbf{0}$ & $\mathbf{0}$ & $\mathbf{0}$ & $\mathbf{0}$ & $\mathbf{0}$ \\
\hline & & \multicolumn{2}{|c|}{ Organization } & 0 & 0 & 0 & 0 & 0 & 0 & 0 & 0 & 0 \\
\hline \multirow{7}{*}{4} & \multirow{7}{*}{ SRL } & \multirow[t]{2}{*}{$\begin{array}{l}\text { Meta- } \\
\text { Cognitive }\end{array}$} & $\begin{array}{l}\text { Volition / } \\
\text { Focus / } \\
\text { Intensity }\end{array}$ & 100 & 100 & 100 & 100 & 100 & 100 & 100 & 100 & 100 \\
\hline & & & Appropriateness & 100 & 100 & 100 & 100 & 100 & 100 & 100 & 100 & 100 \\
\hline & & \multirow{4}{*}{$\begin{array}{l}\text { Affective/ } \\
\text { Motivation }\end{array}$} & $\begin{array}{c}\text { Improving } \\
\text { Pronunciation }\end{array}$ & $<\mathbf{5 0}$ & $<\mathbf{5 0}$ & $<\mathbf{5 0}$ & $<\mathbf{5 0}$ & 100 & 80 & 80 & $<\mathbf{5 0}$ & 80 \\
\hline & & & $\begin{array}{c}\text { Improving } \\
\text { Understanding }\end{array}$ & $<50$ & $<\mathbf{5 0}$ & 80 & $<50$ & 100 & 80 & $<\mathbf{5 0}$ & $<50$ & 100 \\
\hline & & & $\begin{array}{c}\text { Not Clear } \\
\text { Pronunciation for } \\
\text { one word }\end{array}$ & $<\mathbf{5 0}$ & 80 & $<\mathbf{5 0}$ & $<\mathbf{5 0}$ & $<\mathbf{5 0}$ & 100 & $<\mathbf{5 0}$ & $<\mathbf{5 0}$ & $<\mathbf{5 0}$ \\
\hline & & & $\begin{array}{c}\text { Not Clear } \\
\text { Pronunciation for } \\
\text { phrases or } \\
\text { sentences }\end{array}$ & $<\mathbf{5 0}$ & 100 & $<\mathbf{5 0}$ & $<\mathbf{5 0}$ & $<\mathbf{5 0}$ & $<\mathbf{5 0}$ & $<\mathbf{5 0}$ & $<\mathbf{5 0}$ & $<\mathbf{5 0}$ \\
\hline & & \multicolumn{2}{|c|}{ Perseverance / Patience } & $<\mathbf{5 0}$ & $<\mathbf{5 0}$ & $<\mathbf{5 0}$ & $<\mathbf{5 0}$ & 100 & $<\mathbf{5 0}$ & 80 & $<\mathbf{5 0}$ & $<\mathbf{5 0}$ \\
\hline \multirow{2}{*}{5} & & \multirow{2}{*}{\multicolumn{2}{|c|}{$\begin{array}{c}\text { Duration (in Minutes) } \\
\text { Frequency }\end{array}$}} & 80 & 80 & $<\mathbf{5 0}$ & 100 & 100 & 80 & 100 & 80 & 80 \\
\hline & & & & & 80 & & & 100 & & & 100 & \\
\hline
\end{tabular}

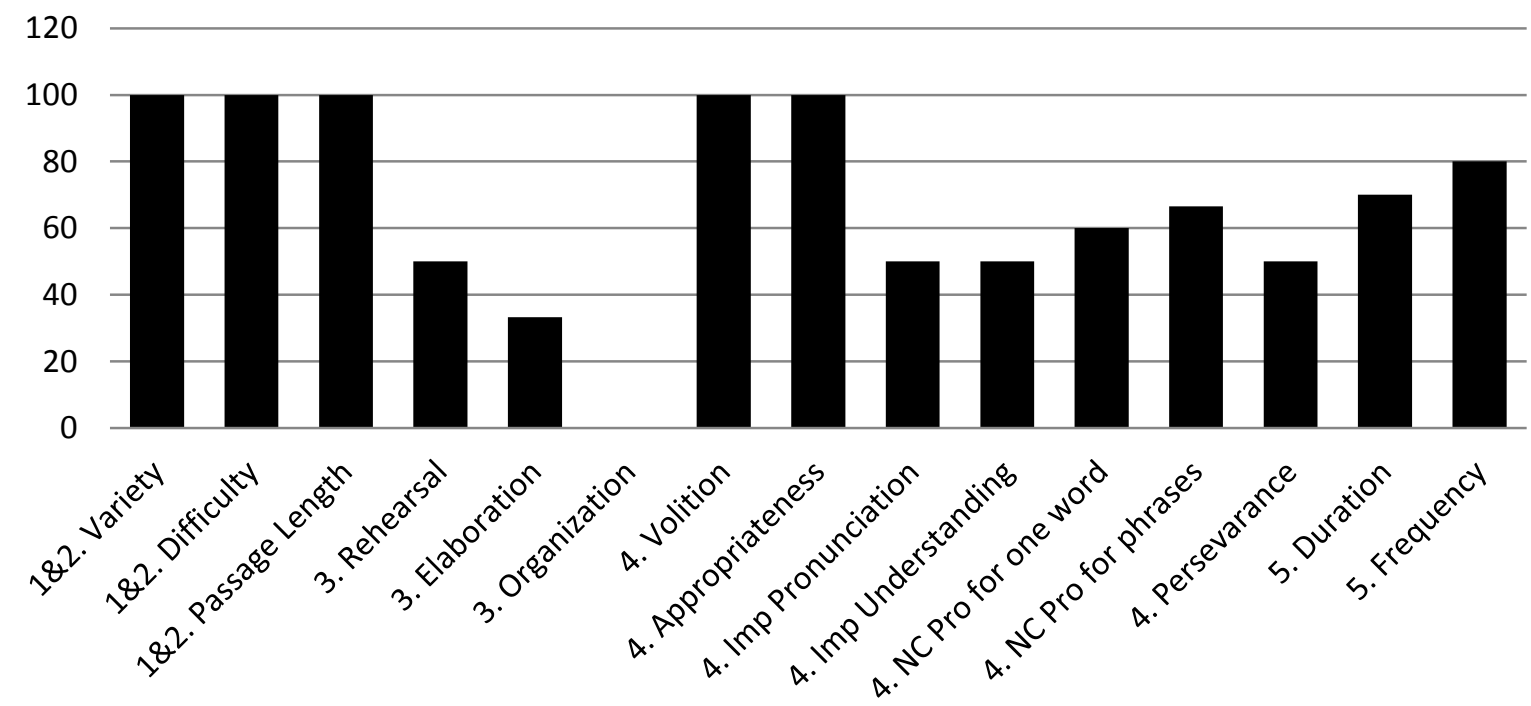

- Figure 3: HSRL Performance of Islam Topic 


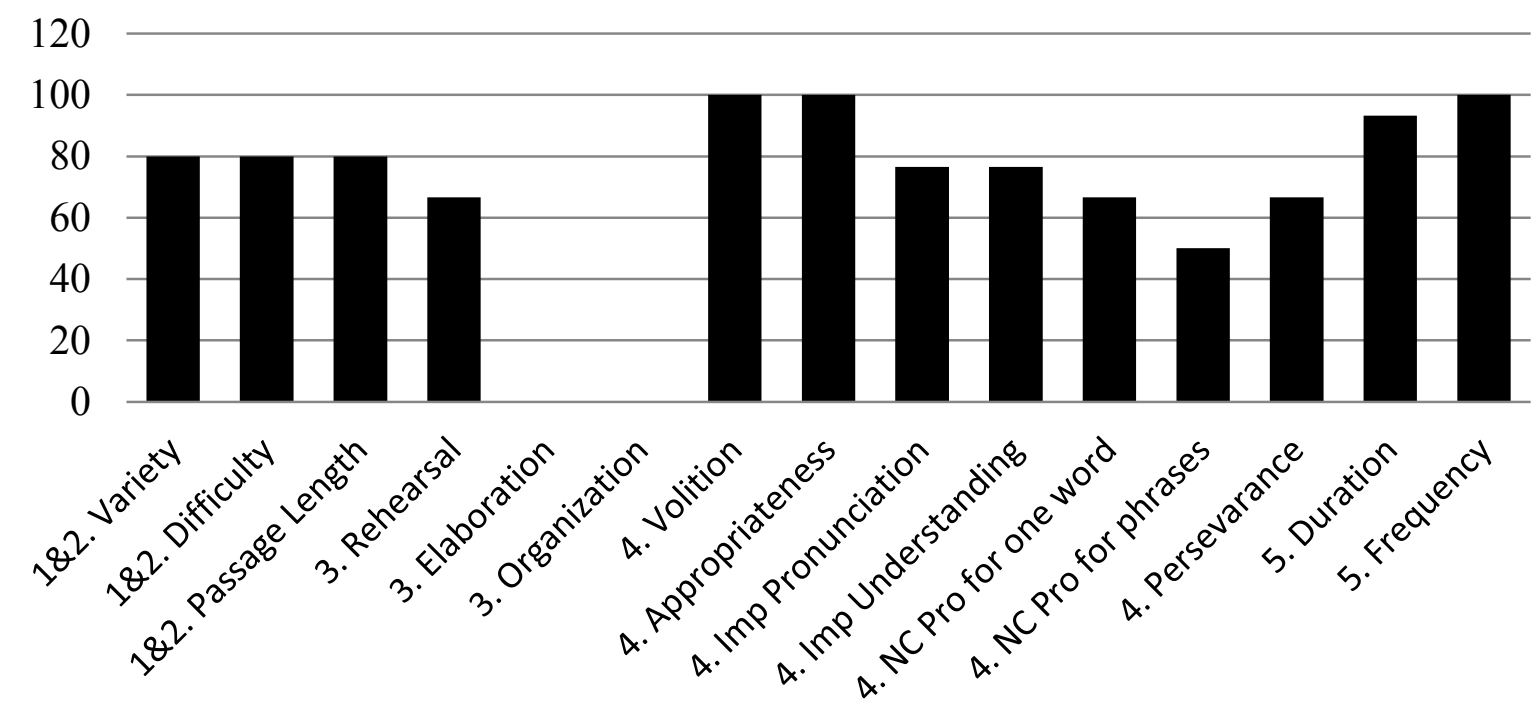

- Figure 4: HSRL Performance of Sport Topic

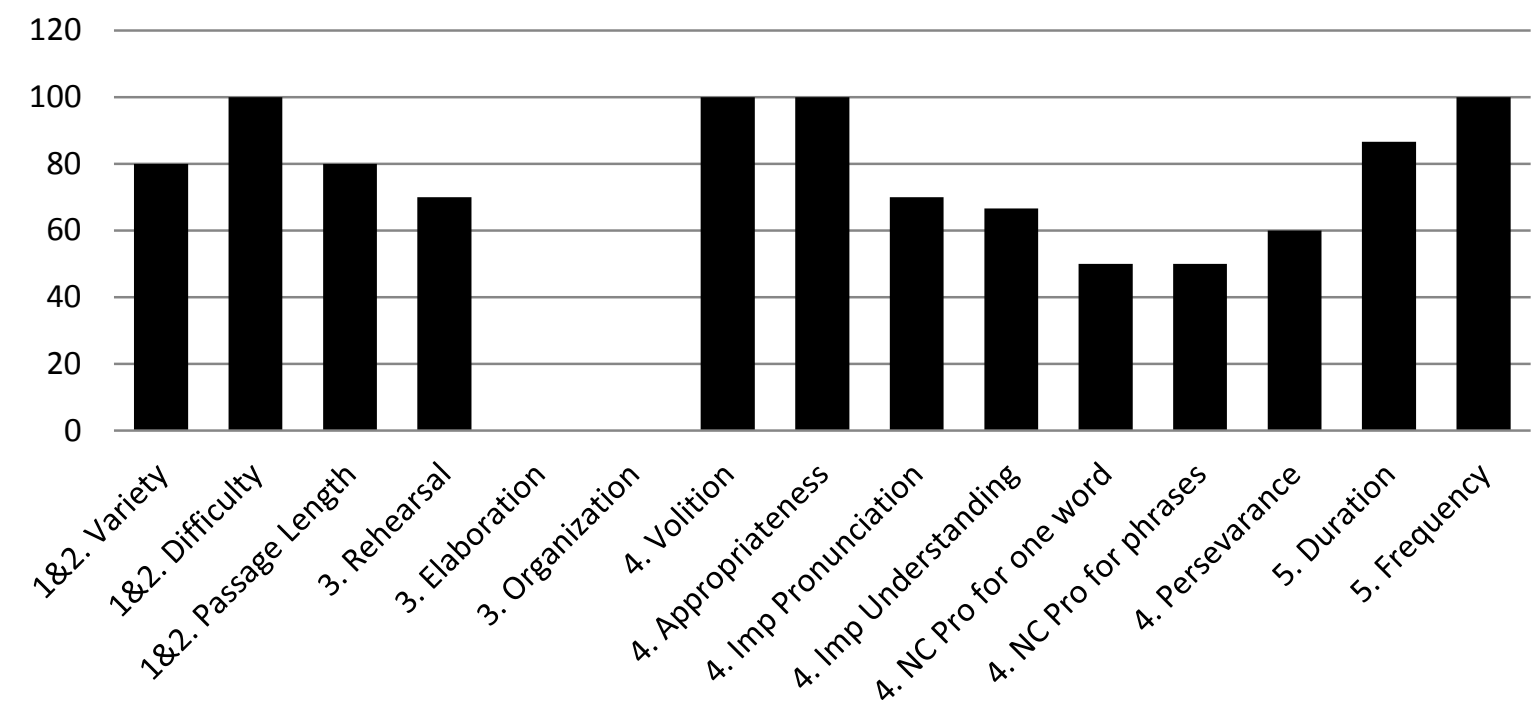

- Figure 5: HSRL Performance of Economics Topic

\subsection{Summary of findings for HSRL}

The student's HSRL by selected topics for beginning, middle and final stages were summarized in Figure (3), Figure (4) and Figure (5). The graphs showed that the medium achievement student employed lower level of use for elaboration and organization at the beginning until the end of the semester. The level of meta-cognition was high in all stages. The student employed elaboration strategy already because she practiced two video resource materials but no organization strategy yet throughout the HSRL practice and feedback treatment. 
Table 2: COP Factors Result Scale

\begin{tabular}{|c|c|c|c|c|c|c|c|c|c|c|c|c|c|}
\hline \multirow{2}{*}{ No } & \multirow{2}{*}{$\begin{array}{c}\text { COP } \\
\text { Evaluated } \\
\text { Factors }\end{array}$} & \multicolumn{6}{|c|}{ Errors } & \multicolumn{6}{|c|}{ Scale } \\
\hline & & Is & An & $\mathrm{Hu}$ & $\mathrm{Sp}$ & $\mathrm{Ag}$ & $\mathrm{Ec}$ & Is & An & $\mathrm{Hu}$ & $\mathrm{Sp}$ & $\mathrm{Ag}$ & Ec \\
\hline 1 & Fluency & 19 & 11 & 49 & $\mathbf{0}$ & 9 & 5 & 15 & 20 & 5 & 20 & 20 & 20 \\
\hline 2 & Pronunciation & 93 & 73 & 64 & 35 & 88 & 40 & 5 & 10 & 10 & 15 & 5 & 15 \\
\hline 3 & Stressing & 20 & 27 & 1 & 9 & 33 & 11 & 20 & 15 & 20 & 20 & 15 & 20 \\
\hline 4 & Intonation & 100 & 100 & 25 & 100 & 100 & 100 & 20 & 20 & 5 & 20 & 20 & 20 \\
\hline 5 & Understanding & 100 & 100 & 50 & 100 & 100 & 100 & 20 & 20 & 10 & 20 & 20 & 20 \\
\hline
\end{tabular}

Note: For fluency, pronunciation and stressing the scoring was conducted based on error reduction while intonation and understanding was scored based on mastery.

25

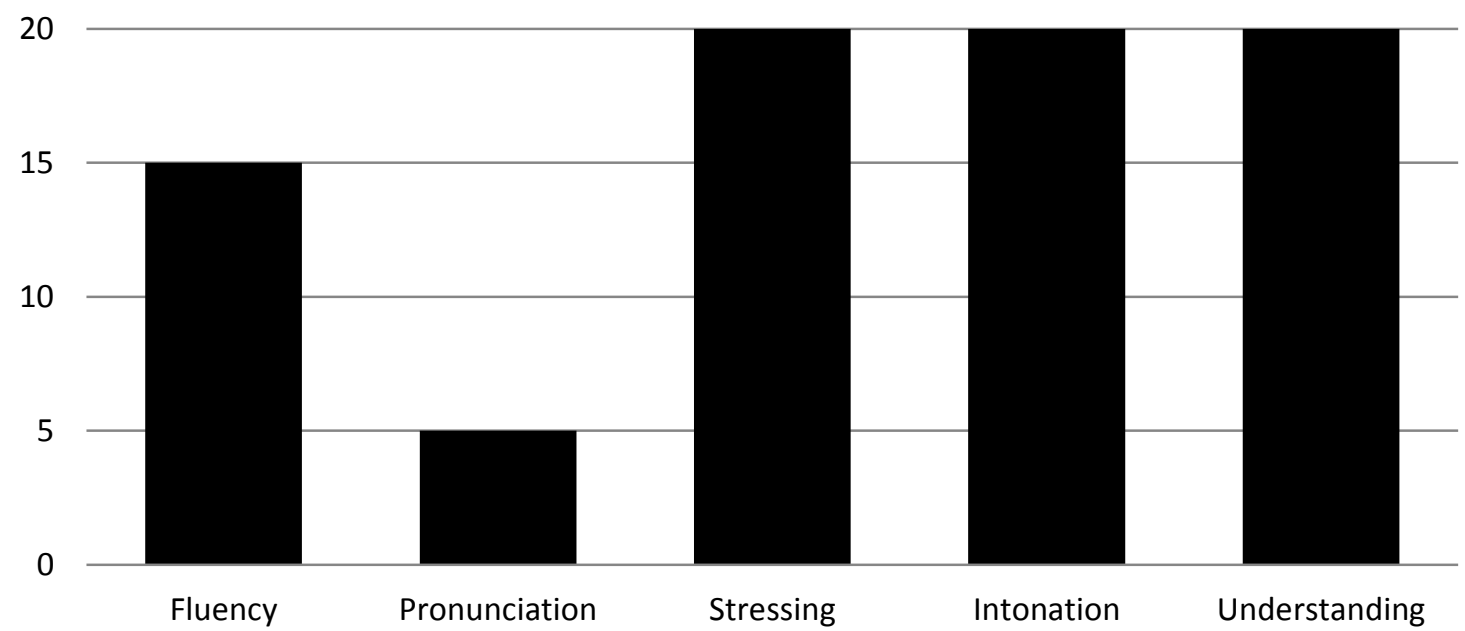

- Figure 6: COP Performance of Islam Topic

25

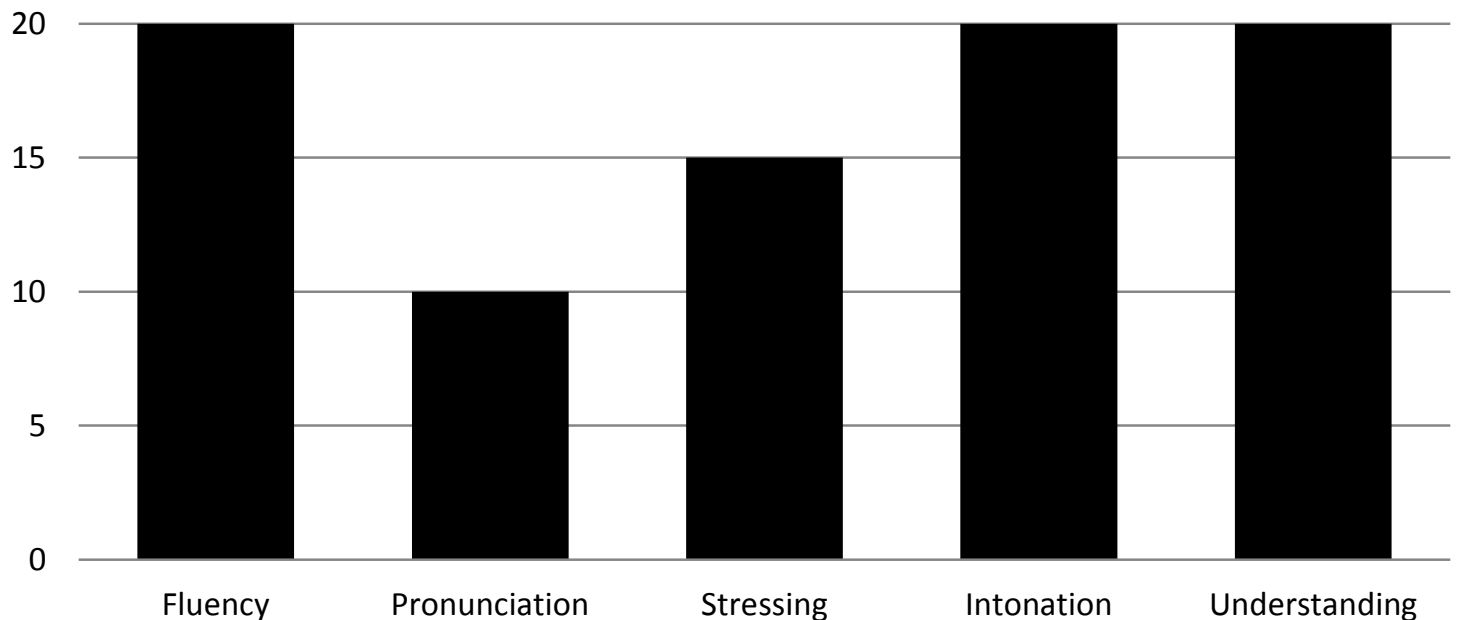

- Figure 7: COP Performance of Animal Topic 
25
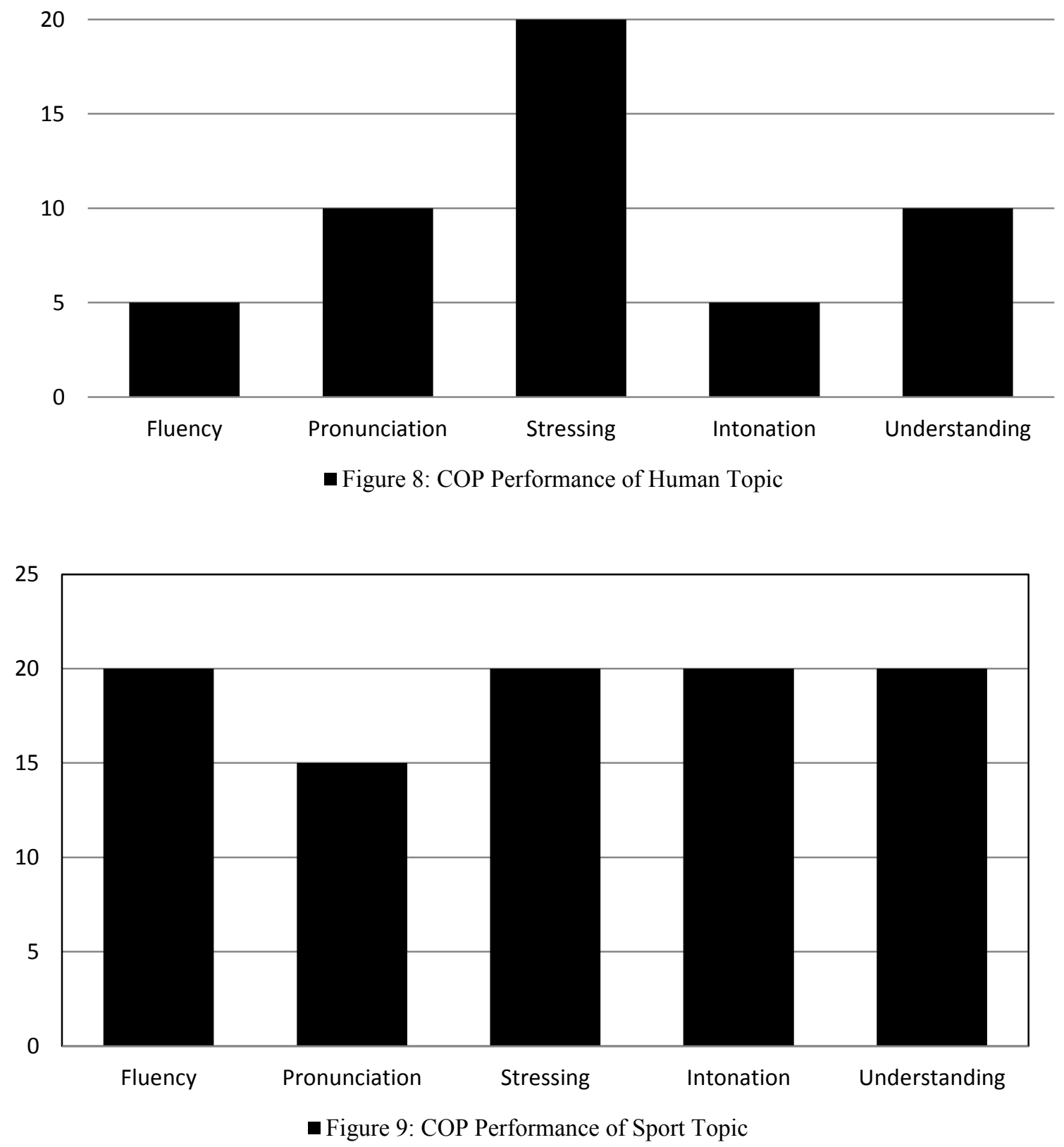
25

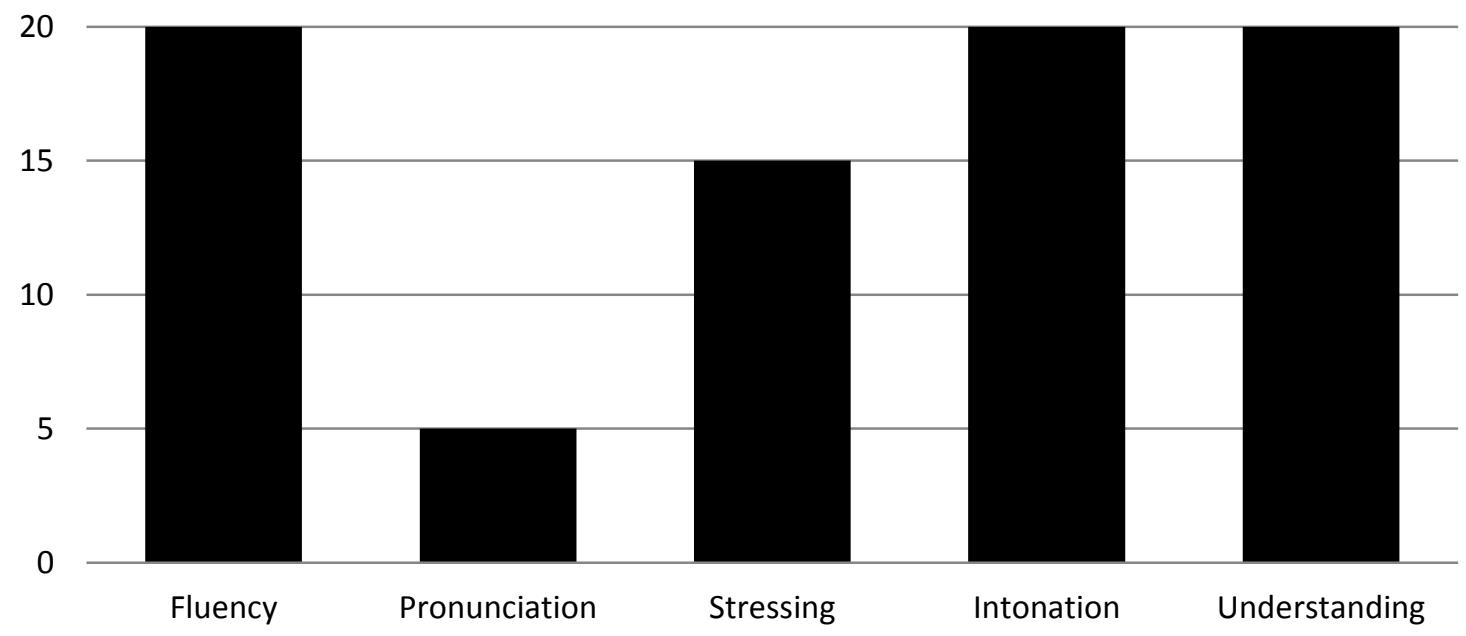

- Figure 10: COP Performance of Agriculture Topic

25

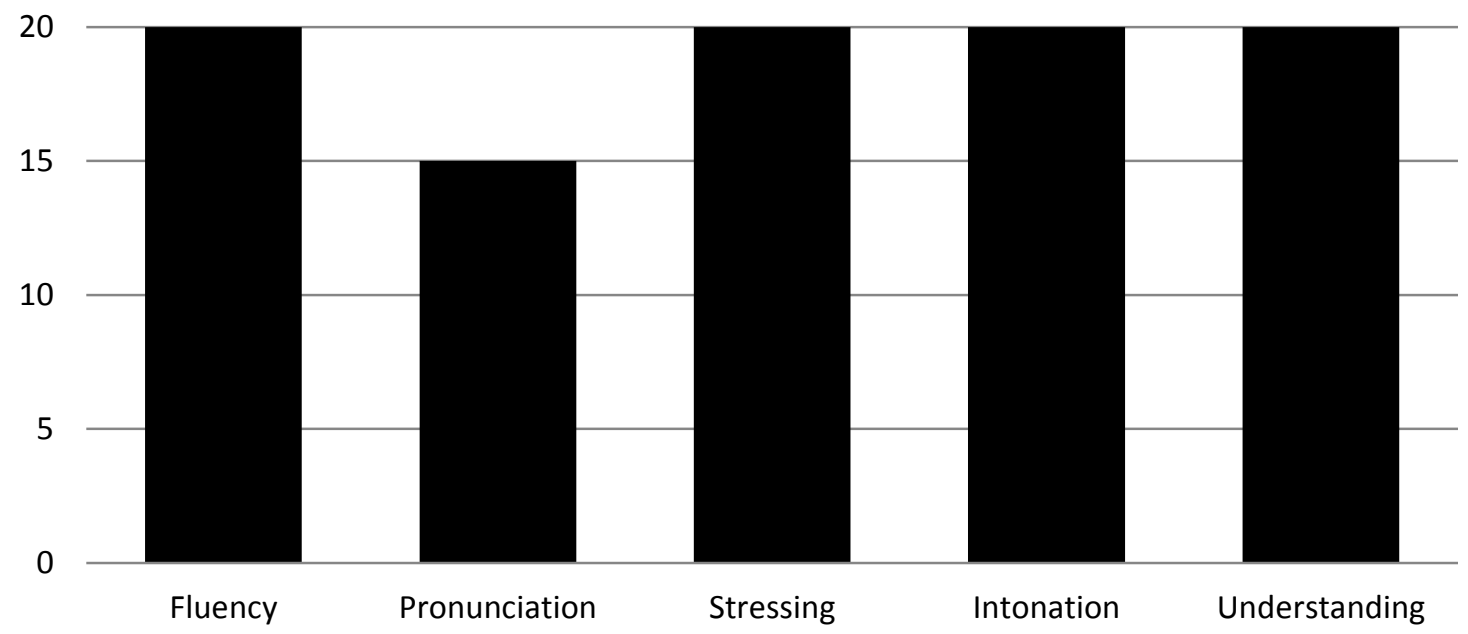

- Figure 11: COP Performance of Economics Topic

\subsection{Summary of findings for COP}

As reported in Table 2 and Figures 6-11, the student had issues in fluency, pronunciation, and stressing with high frequency of errors in the early and middle stages of the experiment (Islam, Animal, Human, \& Agriculture). Over the course of the treatment, these errors were dramatically reduced, especially for Sport topic which was very familiar to the student and good performance was reported for the final COP (Economics).

There was also an inconsistent reduction of errors for topics that contained vocabulary which were new to the student. The improvement of COP performance was contributed by the reduction of errors as well as the acquisition of new knowledge and skills. Oral production was greatly improved after 12 weeks. 


\section{DISCUSSION}

The student was exposed to two conditions as she practiced English pronunciation using NSVC, namely, the instructional condition as prescribed by Nicol and MacFarlane-Dick (2006) to drive the learning cycle based on SRL, and the language learning condition as explained by Levelt (1998) that postulated that the students would deconstruct the inputs to develop lexicons and the capability for internal speech in the L3.

The results proved that the medium achievement student regulated the ranks of use in all HSRL factors such as motivational beliefs, student goals, SRL and duration and frequency of repeats after getting the feedback in each COP except for learning strategy in term of elaboration and organization factors, since rehearsal factor started improving. The graphs showed that she employed lower level of use for elaboration and organization at the beginning until the end of the semester. The level of meta-cognition was high in all stages. These findings were consistent with the prescriptions of Nicol and MacFarlane-Dick (2006). The course goals and standards that were clarified by the instructor initiated a series of actions by the student in selecting the video, setting her goals for each topic, and managing her learning. The feedback after the first oral presentation revealed weaknesses in her performance, and hence, in compensatory move, she increased engagement strategies to overcome them. However, in the final stage, i.e. for the $5^{\text {th }}$ and $6^{\text {th }}$ oral presentations, the engagement levels were reduced slightly as she had received more favourable feedback and felt more confident with the gains achieved.

The instructional condition appeared to be effective in ensuring high levels of commitment and SRL throughout the treatment of the roles of feedback as the mainly influential power on knowledge and attainment (Hattie and Timperley, 2007) obtained by the student during her COP dynamically, personally and supportively. It was given by the instructor smilingly, fairly, patiently and softly with in the presence of her friends that the HSRL language learning cycle was sustained strongly to improve her strategies in her coming HSRL practices to develop her better coming COP.

However, the student employed elaboration strategy already because she had practiced two video resource materials but still she was not able to apply organization strategy at all yet during the practice sessions for the whole of 12 weeks throughout the HSRL practice eventhough she already got the feedback treatment. This finding was consistent with Brown et al (1983) who reported that low or medium ability students did not automatically employ more sophisticated learning strategies unless they were trained or instructed to do so.

The oral production skills showed a marked improvement after 12 weeks. The improvement occurred gradually through the reduction of errors in fluency, pronunciation, and stressing and the increase of understanding and intonation skills. Quick improvement and good performance was shown for topics or words already familiar to the student and tip-of the tongue fluency approximating native-speaker outputs were achieved because the student only had to refine the semantic structures and syntactization of the words. A large number of errors were present for less familiar words and sentences such as in topics on Humans, Agriculture and Economics. This indicated that student was still at the stages of protosyllabary or phonemization of developing the lexical concepts for this topic and two weeks were insufficient for her to achieve tip of the tongue mastery for each set of words.

In general, however, the results showed that persistent and effortful use of the NSVC to buried-deep code-switching trends in the student. The mother tongue language was not inferring with the learning the new words and phrases. The error reduction processes and the rapid acquisition of native-speaker oral qualities showed that the student had gone beyond reproducing phonetic strings and was developing internal speech in English following the steps suggested by Levelt (1998).

\section{CONCLUSION}

The early findings indicated that the NSVC improved oral production in English but more need to be done. Further analysis will compare SRL patterns and performance in oral production between high and low achievement students and also investigate the use of the video clips with peer 
support. The delayed and one-off feedback by the instructor might be too little and too late in enhancing student mastery of oral skills.

\section{REFERENCES}

[1] Ashraf, H., \& Fatemi, M. (2014). The effect of "GO ENGLISH.ME" a virtual learning website on lower intermediate Iranian EFL learners speaking ability. Advances in Language and Literacy Studies, 5(6), 234-238.

http://dx.doi.org/10.7575/aiac.alls.v.5n.6p.234

[2] Brown, A. L., Bransford, J. D., Ferrara, R. A., \& Campione, J. C. (1983). Learning, remembering, and understanding, in J. H. Flavell and E. M. Markman (Eds.). Handbook of Child Psychology, Cognitive Development, (pp. 77-166), New York: Wiley.

[3] Hattie, J., \& Timperley, H. (2007). The power of feedback. Review of Educational Research, 77(1), 81-112. Retrieved from http://rer.sagepub.com/content/77/1/81

[4] Huang, J. (2013). Self-regulation from educational psychology to L2 pedagogy: An alternative to language learning strategies. International Journal of Applied Linguistics \& English Literature, 3(1), 240-244. http://dx.doi.org/10.7575/aiac.ijalel.v.3n.1p.240

[5] Jayakumar, B., \& Rengel, Z. (2009). A model formative assessment strategy to promote student-centered self-regulated learning in higher education. US-China Education Review, 6(12), 29-35.

[6] Levi, D. D. (2005). INTRODUCTION TO RUBRICS An Assessment Tool to Save Grading Time, Convey Effective Feedback, and Promote Student Learning. Virginia: Stylus Publishing.

[7] Min, G. (2006). Vygotsky's sociocultural theory and the role of input and output in second language acquisition. CELEA Journal, 29(4), 88-91.

[8] Nicol, J., \& MacFarlane, D. (2006). Formative assessment and self-regulated learning: A model and seven principles of good feedback practice. Studies in Higher Education, 31(2), 1-19.

[9] Potosi, A., Jhoana, L, Loaiza, G., Andres, E., Garcia, L., \& Catalina, A. (2009). Using video materials as a teaching strategy for listening comprehension. Unpublished research report, Universidad Tecnologica De Pereira.

[10] Sadighi, F., \& Bavali, M. (2008). Chomsky's Universal Grammar and Halliday's Systemic Functional Linguistics: An appraisal and acompromise. Journal of Pan-Pacific Association of Applied Linguistics 12(1), 11-28.

[11] Steiner, C.M., Moore, A., Conlan, O., Dagger, D., Donohoe, G., \& Albert, D. (2013). An investigation of successful self-reguated-learning in a technology-enhanced learning environment. Proceedings of the AIED 2013 Conference, 19-23.

[12] Ullman, M. (2001). The Declarative/Procedural Model of lexicon and grammar. Journal of Psycholinguistic Research, 30 (1), 37-69.

[13] Watkins, J., \& Wilkins, M. (2001). Using Youtube in EFL clasroom. Language Education in Asia, 2(1), 113-119. http://dx.doi.org/10.5746/LEiA/11/V2/I1/A09/Watkins_Wilkins 